\title{
Erythrocytes' osmotic fragility test with a standard Abbe refractometer
}

\author{
Argelia Pérez-Pacheco ${ }^{1}$, Laura B. Salazar-Nieva², Augusto García-Valenzuela ${ }^{3}$, \\ Emma R. Mendoza-García ${ }^{4}$, Adolfo Martínez-Tovar ${ }^{5}$, Irma Olarte-Carrillo ${ }^{5}$, and Roberto Márquez-Islas ${ }^{1 *}$ \\ ${ }^{1}$ Unidad de Investigación y Desarrollo Tecnológico, Hospital General de México "Dr. Eduardo Liceaga"; ${ }^{2}$ Facultad de Ciencias, Universidad Nacional \\ Autónoma de México; ${ }^{3}$ Instituto de Ciencias Aplicadas y Tecnología, Universidad Nacional Autónoma de México; ${ }^{4}$ Laboratorio de Hematología \\ Especial, Hospital General de México, "Dr. Eduardo Liceaga"; ${ }^{5}$ Laboratorio de Biología Molecular, Hospital General de México, "Dr. Eduardo Liceaga". \\ Mexico City, Mexico
}

\begin{abstract}
Background: The osmotic fragility (OF) test is one of the most suitable procedures to determine the resistance of the erythrocyte membrane under conditions of osmotic stress. One of the most frequent clinical uses of the FO test is the diagnosis of hereditary conditions that affect erythrocytes: thalassemia and spherocytosis. Both conditions induce red blood cells (RBCs) to be prone to swelling and then lyse under osmotic stress faster than those RBCs without any pathology. Since the standard OF test is time consuming and needs specialized equipment, a fast, accessible, and reliable technique is necessary to determine the OF Objective: The objective of the study was to evaluate an alternative technique to determine the OF of erythrocytes by refractive index (RI) measurements. The method consists of measuring the real part of the $R I$ of dilutions of whole blood samples with hypotonic solutions at different $\mathrm{NaCl}$ concentrations, ranging from $0 \%$ to $0.9 \%$ using a standard Abbe refractometer. Materials and methods: An experimental study was carried out with volunteers who went to the General Hospital of Mexico "Eduardo Liceaga." Peripheral blood samples from 12 healthy volunteers were used through the standard blood draw procedure and stored in a heparinized vacutainer tube. Results and conclusions: The proposed technique that uses an Abbe refractometer to measure the infrared of different solutions of whole blood and hypotonic solutions to determine the OF curve has proven to be simpler and more robust than the standard method based on the measurement of light absorption in dilutions of hemolyzed blood. The determination of the OF of erythrocytes rapidly and straightforwardly can be useful in the detection of specific pathologies.
\end{abstract}

Key words: Osmotic fragility. Erythrocyte membrane. Refractometry. Refractive index.

\section{Introduction}

The erythrocytes, commonly known as red blood cells (or RBCs), are the most numerous cells in the blood, and their main function is to transport oxygen to the different tissues in the human body and collect $\mathrm{CO}_{2}$ to be discarded. RBCs are composed by a thin semi-permeable membrane and are extremely flexible since it is continually forced to slide through blood vessels of different diameters, even smaller than the diameter of an average erythrocyte ${ }^{1}$. This mechanical stress is well tolerated under normal conditions by most of the RBC. The external membrane is permeable to different substances: water, oxygen, carbon dioxide, etc., but is impermeable to the hemoglobin which is one of its main

\section{Correspondence:}

*Roberto Márquez-Islas

E-mail: roberto.marquez@icat.unam.mx
Available online: 17-01-2020

Date of reception: 21-12-2018

DOI: 10.24875/HGMX.19000012
Rev Med Hosp Gen Mex. 2020;83(1):20-25 www.hospitalgeneral.mx 0185-1063/@ 2019 Sociedad Médica del Hospital General de Mexico. Published by Permanyer. This is an open access article under the CC BYNC-ND license (http://creativecommons.org/licenses/by-nc-nd/4.0/). 
internal constituents. Under isotonic conditions $(0.9 \%$ solution of $\mathrm{NaCl}$ ), this membrane is in osmotic equilibrium with the aqueous solution in which they are suspended (plasma) and regulate the balanced exchange of substances between the erythrocyte and the plasma, allowing volumes of nutrients enter and leave the cell without significant changes in the volume or physical properties. The erythrocytes are physically biconcave disc-shaped structures containing no nucleus; they have a diameter of approximately $7.5 \mu \mathrm{m}$, a thickness of approximately $2 \mu \mathrm{m}$ and an expected lifetime of $100-120$ days $^{2}$.

As mentioned above, an erythrocyte in its daily function is under different types of stress; however, this flexibility can be affected by a great variety of factors, such as the presence of some hemolytic pathologies or the effect of some drugs. Consequently, the membrane may become more fragile, and the erythrocytes' integrity may be compromised. The capacity of erythrocytes to resist the rupture of their membranes that produce the expulsion of the intracellular material to the plasma is called the membrane's osmotic fragility $(\mathrm{OF})^{3}$.

The OF test is one of the oldest methods to study the physical state of erythrocyte ${ }^{4}$, among other factors, gives us information about their health status. Its use to determine the resistance of erythrocytes was initially described by Parpart and Lorenz in $1947^{5}$. The test's procedure seeks to quantify the percentage of erythrocytes' lysis in a blood sample under osmotic stress conditions by means of mixing it with $\mathrm{NaCl}$ solutions of decreasing concentration, ranging from $0.9 \%$ to $0 \%$ (hypotonic solutions). The hemolysis quantification in percentage plotted versus $\mathrm{NaCl}$ concentration is called the OF curve. Any shift of the OF curve to low or high concentrations of $\mathrm{NaCl}$ with respect to healthy $\mathrm{RBC}$ may indicate the presence of any alteration or a pathology ${ }^{4,6,7}$. In the standard method, the percentage of hemolyzed erythrocytes in a suspension is determined by measuring the absorption coefficient of the medium surrounding the erythrocytes. It is required to remove all cells from suspension, whether their membrane is ruptured or not, and then measure the extinction coefficient of light passing the remaining homogeneous liquid $^{6}$. The procedure is time consuming, and measurements of the extinction coefficient are prone to many errors, thus require skilled personnel to perform accurate readings.

In this work, we present an easy-to-implement method to quantify the degree of hemolysis of a blood sample by measuring the refractive index (RI) of whole

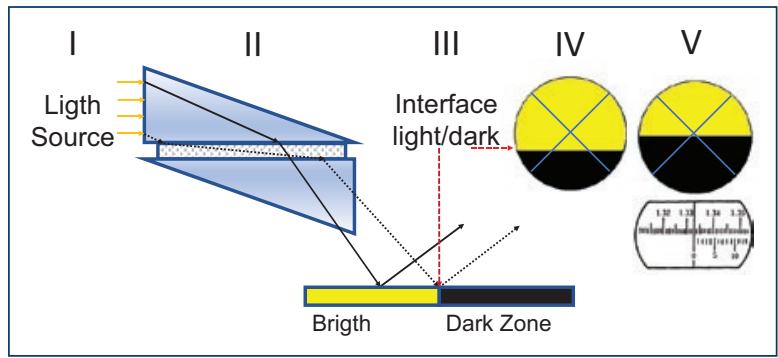

Figure 1. Abbe refractometer scheme.

blood dilutions using an Abbe refractometer. Depending on the saline concentration of the solution used to dilute the blood, a percentage of erythrocytes will swell, and burst, releasing its content into the plasma (being the hemoglobin the main component) and changing the sample's RI directly proportional to the percentage of hemolysis.

The Rl describes how light propagates through a medium and is defined as the ratio of the speed with which a light beam propagates in an unknown medium $\left(v_{m}\right)$ to that in a vacuum $\left(v_{0}\right)$, that is, $n=v_{m} / v_{0}$. Optical refractometry is a basic tool for pharmaceutical, food, chemical, and oil industries, as well as in many research laboratories ${ }^{8-12}$. The Abbe refractometer is a well-established optical device that measures directly and instantly the RI of transparent or translucent liquids. It is a robust instrument that can be used outside the laboratory and has a relatively low cost. It does not require expensive maintenance.

The Abbe refractometer consists of two prisms, the upper prism is a diffuser and the lower prism has a higher $\mathrm{RI}(\mathrm{n} \approx 1.7)$. The sample to be analyzed is placed between the two prisms forming a uniform film of approximately 100 microns wide (II in Fig. 1). When illuminated with a light source (I in Fig. 1), the diffuser prism scatter light in all directions. The scattered rays travel the full width of the sample. All scattered rays reaching the lower prism refract inside the lower prism at angles of refraction up to the critical angle defined by the $\mathrm{RI}$ of the lower prism and the sample. The rays refracted at the critical angle correspond to rays traveling parallel to the prisms' interfaces. No light can be refracted at angles of refraction higher than the critical angle. Hence, a sharp light/dark angular border is formed at the critical angle when viewing the lower-prism/sample interface (III in Fig. 1). This angular border can be directly observed by an operator using suitable optics, and its location in an appropriate angular scale gives the RI if the sample (IV-V in Fig. 1). 
A common uncertainty in $\mathrm{RI}$ measurements in inexpensive Abbe refractometers is \pm 0.0002 .

\section{Materials and methods}

We first prepared ten hypotonic samples (HS) by diluting a saline solution of $0.9 \% \mathrm{w} / \mathrm{v}$ of $\mathrm{NaCl}$ with tri-distilled water, decreasing in approximately $10 \%$ the concentration of $\mathrm{NaCl}$ with each dilution. The resulting samples (including isotonic sample) are shaken gently to make the mixture homogeneous. Then, we measured the Rl of each sample using the Abbe refractometer. Table 1 shows RI values measured from tri-distilled water (M0) to the isotonic serum (M10) (Table 1).

The theoretical RI values of HS were calculated by the volume-weighted rule formula for the RI of miscible mixtures, that is,

$$
n=f_{\mathrm{v}} \cdot 1.3327+\left(1-f_{\mathrm{v}}\right) \cdot 1.3345
$$

Where $f_{v}$ is the volume fraction of tri-distilled water, $1-f_{v}$ is the saline solution volume fraction and 1.3327, 1.3345 the $\mathrm{RI}$ of tri-distilled water, and the $0.9 \% \mathrm{w} / \mathrm{V}$ $\mathrm{NaCl} /$ water solution, respectively, measured with the Abbe refractometer. In figure 2, the bars (gray line) represent the error associated with the measurements made with the Abbe ( \pm 0.0002) (Fig. 2).

Peripheral blood samples obtained by venipuncture of nonhospitalized and healthy volunteers were obtained through the standard procedure of blood extraction and stored in a heparinized vacutainer tube. To obtain the curve of the RI of blood dilutions versus the $\mathrm{NaCl}$ concentration, we mixed a volume of $0.2 \mathrm{ml}$ of whole blood and $0.8 \mathrm{ml}$ of one of the hypotonic solutions, at this volume ratio $(20-80 \%$, respectively). The changes in RI due to the release of hemoglobin by the hemolysis in the samples are large enough to be measured by the Abbe refractometer. The values for the tri-distilled water (M0) and the isotonic serum (M10) are taken as the reference values for $100 \%$ and the $0 \%$ hemolysis, respectively. The whole blood is added to the HS and mixed homogeneously by reabsorbing the sample gently 2 times with a micropipette and then leave the sample to rest for $6 \mathrm{~min}$. Finalizing this time, we measure the RI with an Abbe refractometer 2WAJ (at $\lambda=589 \mathrm{~nm}$ ). This process is repeated for all HS, including the isotonic solution.

\section{Results}

In figure $3 \mathrm{~A}-\mathrm{L}$, we show the RI of the blood dilutions as a function of the $\mathrm{NaCl}$ concentration from the 12
Table 1. Refractive index (calculated and measured with Abbe refractometer) of $\mathrm{NaCl}$ and tri-distilled water solutions at different concentrations

\begin{tabular}{|l|c|c|c|}
\hline Sample & Nacl (\%) & RI (calculated) & RI Abbe $( \pm \mathbf{0 . 0 0 0 2})$ \\
\hline M0 & 0 & 1.3327 & 1.3329 \\
\hline M1 & 0.09 & 1.3328 & 1.3332 \\
\hline M2 & 0.18 & 1.3330 & 1.3334 \\
\hline M3 & 0.27 & 1.3332 & 1.3335 \\
\hline M4 & 0.36 & 1.3334 & 1.3337 \\
\hline M5 & 0.45 & 1.3336 & 1.3338 \\
\hline M6 & 0.54 & 1.3337 & 1.3348 \\
\hline M7 & 0.63 & 1.3339 & 1.3342 \\
\hline M8 & 0.72 & 1.3341 & 1.3340 \\
\hline M9 & 0.81 & 1.3343 & 1.3344 \\
\hline M10 & 0.9 & 1.3345 & 1.3345 \\
\hline
\end{tabular}

$\mathrm{Rl}$ : refractive index.

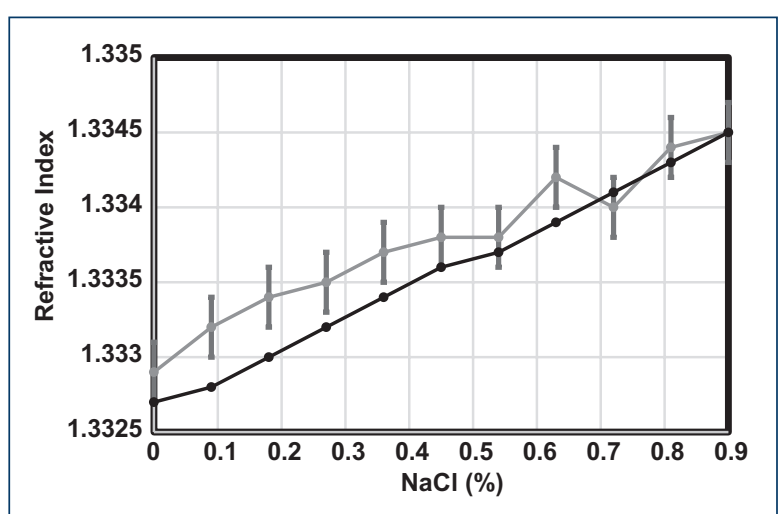

Figure 2. Graph of refractive index (RI) of M0-M10 samples measured with an Abbe refractometer (gray line), and the RI calculated by 1 (black line).

volunteers. The variations in the curves for healthy volunteers can be explained due to the age, sex, and genetic diversity of the donors' population. These factors modulate the characteristics of RBCs that result in a difference in the process of recovery of erythrocytes under different stress conditions and shows a different hemolysis process ${ }^{13}$. A more detailed analysis of these differences is left for later work (Fig. 3).

In figure 4A (black line), we plot the RI average curve from the blood samples as a function of the salt concentration, the bars represent the standard deviation from the experimental data. For comparison, in figure 4B, 


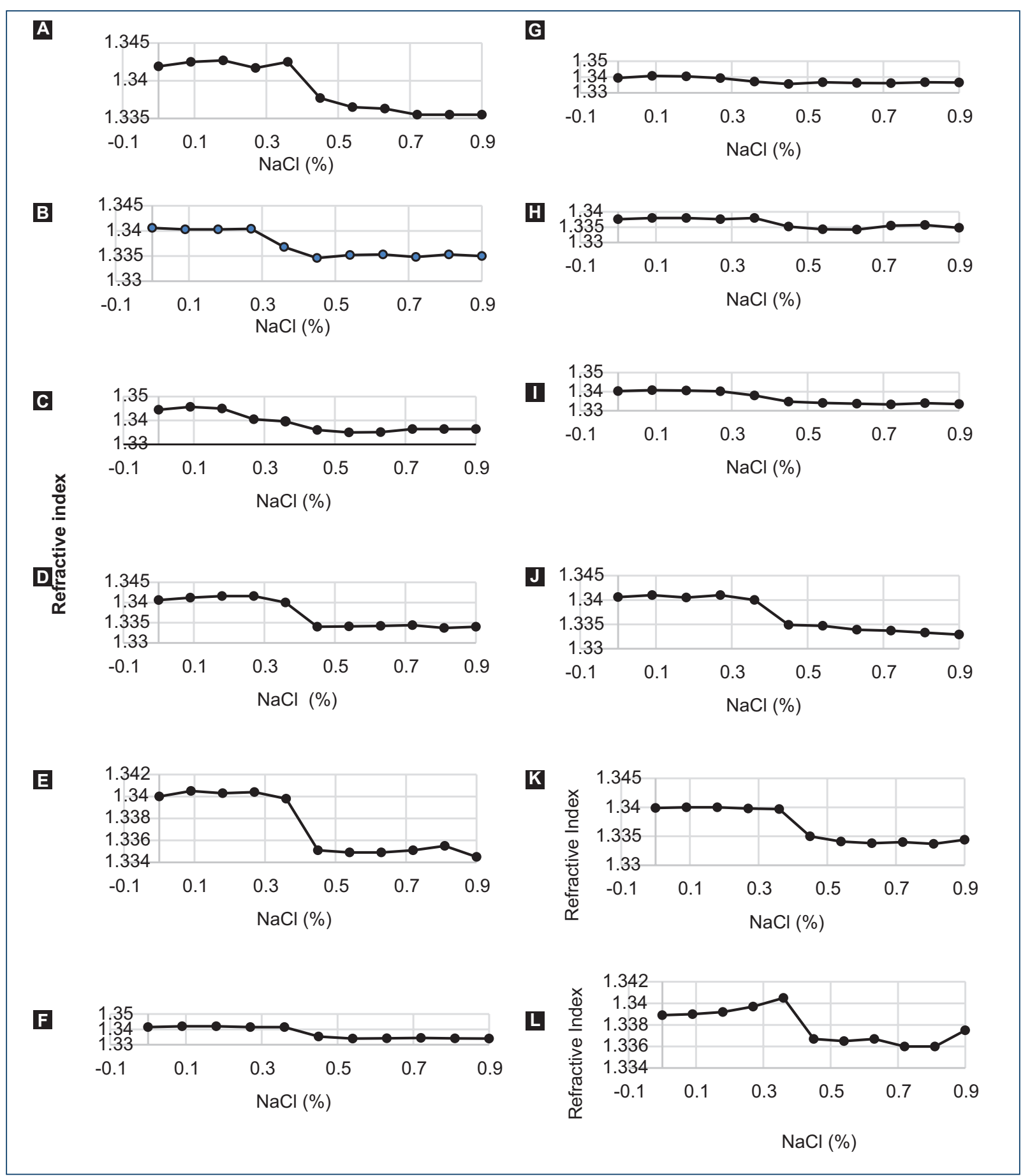

Figure 3. Refractive index versus hypotonic samples from 12 volunteers.

the OF curve (gray line) is expressed in percentage of hemolysis from experimental data obtained from five healthy volunteers six using the standard procedure: measuring the absorbance (at $540 \mathrm{~nm}$ wavelength) of blood dilutions in hypotonic media after removing the remaining erythrocytes, and then calculating the percentage of lysis ${ }^{14}$ (Fig. 4).
In figure $4 \mathrm{~A}$, we can appreciate that from $0.0 \%$ to $0.36 \% \mathrm{w} / \mathrm{V}$ concentration of $\mathrm{NaCl}$, the $\mathrm{RI}$ average value obtained in this work is about $1.3403 \pm 3.0 \times 10^{-3}$. For these salt concentrations, hemolysis is complete, and most of the hemoglobin of the erythrocytes is dissolved in the plasma. In these cases, the borderline that serves to determine the value of the $\mathrm{RI}$ in the Abbe refractometer 


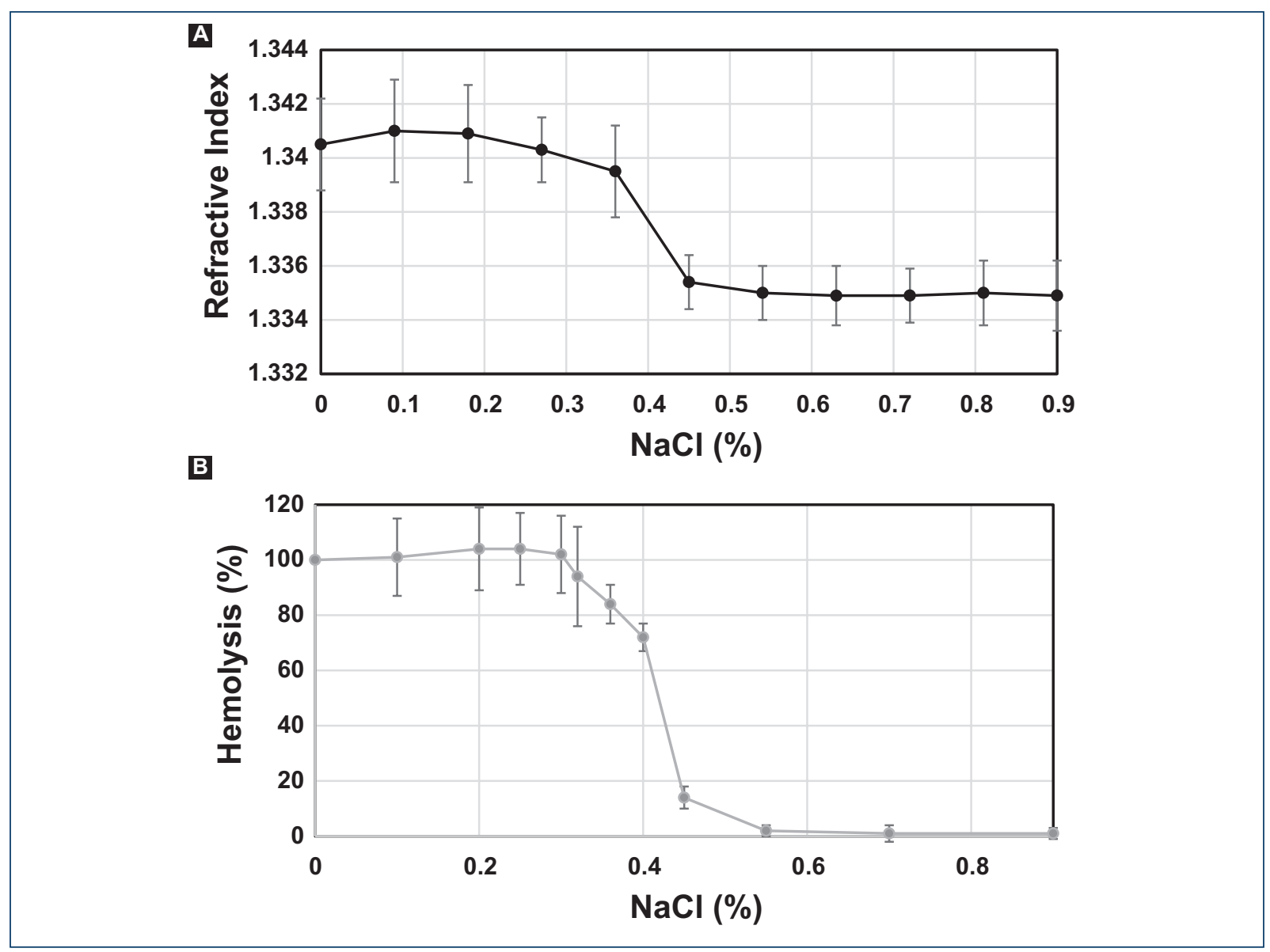

Figure 4. Comparison between the curves of the refractive index. A: percentage of hemolyzed blood. B: at different hypotonic $\mathrm{NaCl}$ concentrations. A: the bars in the average curve show the standard deviation (SD) that was obtained were obtained from the 12 experimental data. B: its respective SD was reported in reference ${ }^{6}$.

(Fig. $5 \mathrm{~A}$ ) is very sharp and easy to read. This behavior changes to concentrations of 0.36 and higher, where the value of RI falls for all samples. This can be understood from the fact that the percentage of ruptured erythrocytes is lower because the concentration of $\mathrm{NaCl}$ is approaching the isotonic solution. Then, the number of erythrocytes that remain complete increases and tends to settle on the refractometer lower prism. These full erythrocytes scatter light and the borderline that serves as a reference in the Abbe becomes diffuse as time passes (Fig. 5B), so measurements should be done immediately after depositing the sample to be able to appreciate a borderline as sharp as possible and record the correct Rl's value, for this reason, the measurements were repeated two times for concentrations of $0.45 \mathrm{NaCl}$ and higher. After each measurement, we used tri-distilled water to avoid contamination and prepare the refractometer for the next measurement (Fig. 5).

We can appreciate in figure 4 that both experimental curves for the $\mathrm{RI}$ and the percentage of hemolysis obtained by the standard procedure are qualitatively very similar in their behavior ${ }^{4,6}$. It shows clearly that for high levels of hemolysis in the suspension, the RI is maximum and as the percentage of lysis decreases, the $\mathrm{RI}$ is lower.

\section{Discussion and conclusions}

We found that despite the optical absorption and scattering of light in whole blood dilutions in hypotonic solutions, it is possible to measure the RI of the dilutions using a standard Abbe refractometer. The ratio of $20-80 \%$ of whole blood HS dilutions was found to be the optimal concentration for measurements in our Abbe refractometer to measure the change in the samples' RI due to the hemoglobin released in the plasma.

We obtained experimental measurements of the RI of whole blood dilutions in hypotonic solutions from 12 healthy volunteers using a commercial Abbe 


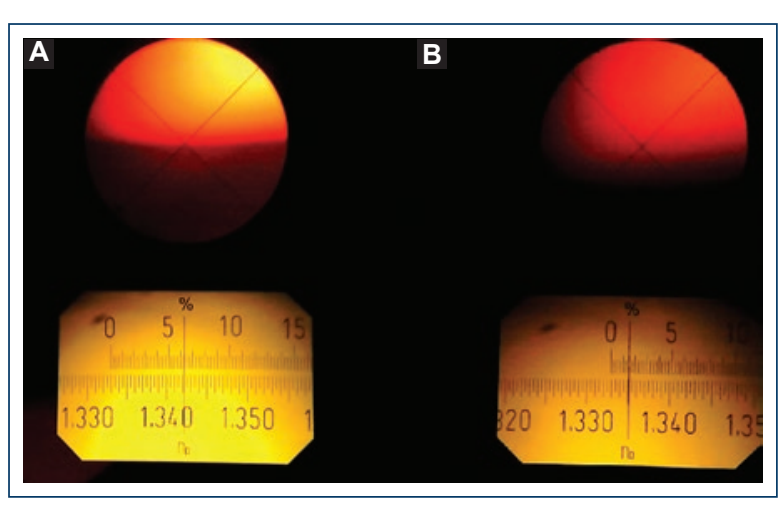

Figure 5. A: borderline, as seen in the Abbe refractometer for high hemolyzed blood samples. B: borderline for samples with a low percentage of hemolysis (diffuse).

refractometer (2WAJ). From the data obtained, we present curves for each volunteer, and the average curve is compared with the experimental results for OF curve presented in Alonso-Geli et al. ${ }^{6}$ Both average curves show similar behavior and coincide to determine that hemolysis for healthy volunteers starts at $0.45-0.36$ of $\mathrm{NaCl}$. We found from these measurements that the mean value of the infrared of high hemolyzed blood samples is $1.3403 \pm 3.0 \times{ }^{-3}$ and non-hemolyzed blood is $1.3350 \pm 0.001$.

In summary, we illustrate in this work the viability of simple and rapid procedure to determine the standard OF curve of blood samples and provides information related to the integrity of the erythrocyte membrane, which can be an indicator of the health status of people associated with different pathologies such as: thalassemia $^{15,16}$, macro or micro spherocytosis ${ }^{17,18}$, and paroxysmal nocturnal hemoglobinuria ${ }^{19,20}$, among others ${ }^{21}$. One of its main advantages over other techniques such as flow cytometry is that it is a rapid and economical test so that it does not require large material resources and can open the way to develop new robust instruments for the early detection of different blood pathologies.

\section{Acknowledgments}

We acknowledge funding from Dirección General de Asuntos del Personal Académico from Universidad Nacional Autónoma de México through Project IN-102218. We also want to acknowledge the invaluable help from the Blood Bank at Hospital General de México "Dr. Eduardo Liceaga."

\section{Conflicts of interest}

The authors declare that they have no conflicts of interest.

\section{Ethical disclosures}

Protection of human and animal subjects. The authors declare that no experiments were performed on humans or animals for this study.

Confidentiality of data. The authors declare that no patient data appear in this article.

Right to privacy and informed consent. The authors declare that no patient data appear in this article.

\section{References}

1. Rogers K. Blood: physiology and Circulation. New York: Britannica Educational Publishing, European University Association; 2011.

2. Schaller J. Human Blood Plasma Proteins: structure and Function. Hoboken, New Jersey: John Wiley and Sons Ltd.; 2008.

3. Mazeron P, Didelon J, Muller S, Stoltz JF. A theoretical approach of the measurement of osmotic fragility of erythrocytes by optical transmission. Photochem Photobiol. 2000;72:172-8.

4. Beutler E, Kuhl W, West C. The osmotic fragility of erythrocytes after prolonged liquid storage and after reinfusion. Blood. 1982;59:1141-7.

5. Parpart AK, Lorenz PB, Parpart ER, Gregg JR, Chase AM. The osmotic resistance (fragility) of human red cells. J Clin Invest. 1947;26:636-40.

6. Alonso-Geli Y, Alonso-Moreno Y, Falcón-Diéguez LE, Lucambio-Miró EL, Castro-Piñol EM. Caracterización de la fragilidad osmótica de eritrocitos humanos en la anemia drepanocítica. Rev Cuba Quím. 2015;27:110-8. Available from: http://www.scielo.sld.cu/pdf/ind/v27n2/ind01215.pdf.

7. Sprandel U, Zöllner N. Osmotic fragility of drug carrier erythrocytes. Res Exp Med (Berl). 1985;185:77-85.

8. Rheims J, Köser J, Wriedt T. Refractive-index measurements in the nearIR using an Abbe refractometer. Meas Sci Technol. 1997;8:601-5.

9. Kavitha S, Murthy VR. Refractometry as a tool in diabetic studies. Anc Sci Life. 2006;25:76-83.

10. Oliveira L, Lage A, Pais Clemente M, Tuchin V. Optical characterization and composition of abdominal wall muscle from rat. Opt Lasers Eng. 2009;47:667-72.

11. Possetti GR, Kamikawachi RC, Prevedello CL, Muller M, Fabris JL. Salinity measurement in water environment with a long period grating based interferometer. Meas Sci Technol. 2009;20:034003.

12. Li J, Wen CH, Gauza S, Lu R, Wu ST. Refractive indices of liquid crystals for display applications. J Disp Technol. 2005;1:51-61.

13. Kanias T, Lanteri MC, Page GP, Guo Y, Endres SM, Stone M, et al. Ethnicity, sex, and age are determinants of red blood cell storage and stress hemolysis: results of the REDS-III RBC-Omics study. Blood Adv. 2017;1:1132-41.

14. Walski T, Chludzi『ska L, Komorowska M, Witkiewicz W. Individual osmotic fragility distribution: a new parameter for determination of the osmotic properties of human red blood cells. Biomed Res Int. 2014;2014:162102.

15. Maccioni L, Cao A. Osmotic fragility test in heterozygotes for alpha and beta thalassaemia. J Med Genet. 1985;22:374-6.

16. Sears DA, Udden MM, Johnston MD. Red cell osmotic fragility studies in hemoglobin C-beta thalassemia: osmotically resistant microspherocytes. Clin Lab Haematol. 2003;25:367-72.

17. Hassan A, Babadoko AA, Isa AH, Abunimye P. Hereditary spherocytosis in a 27-year-old woman: case report. Ann Afr Med. 2009;8:61-3.

18. Emilse LA, Cecilia H, María TM, Eugenia MM, Alicia IB, Lazarte SS. Cryohemolysis, erythrocyte osmotic fragility, and supplementary hematimetric indices in the diagnosis of hereditary spherocytosis. Blood Res. 2018;53:10-7.

19. Mohammed AA, El-Tanni H, Atiah TA, Atiah AA, Atiah MA, Rasmy AA. Paroxysmal nocturnal hemoglobinuria: from bench to bed. Indian $\mathrm{J} \mathrm{He}$ matol Blood Transfus. 2016;32:383-91.

20. Parker CJ. Paroxysmal nocturnal hemoglobinuria: an historical overview. Hematology Am Soc Hematol Educ Program. 2008;(1):93-103.

21. Márquez-Islas R, Pérez-Pacheco A, Salazar-Nieva LB, Acevedo-Barrera A, Mendoza-García E, García-Valenzuela A. Optical Device and Methodology for Optical Sensing of Hemolysis in Hypotonic Media. Meas. Sci. Technol. 2019. Article under review. 